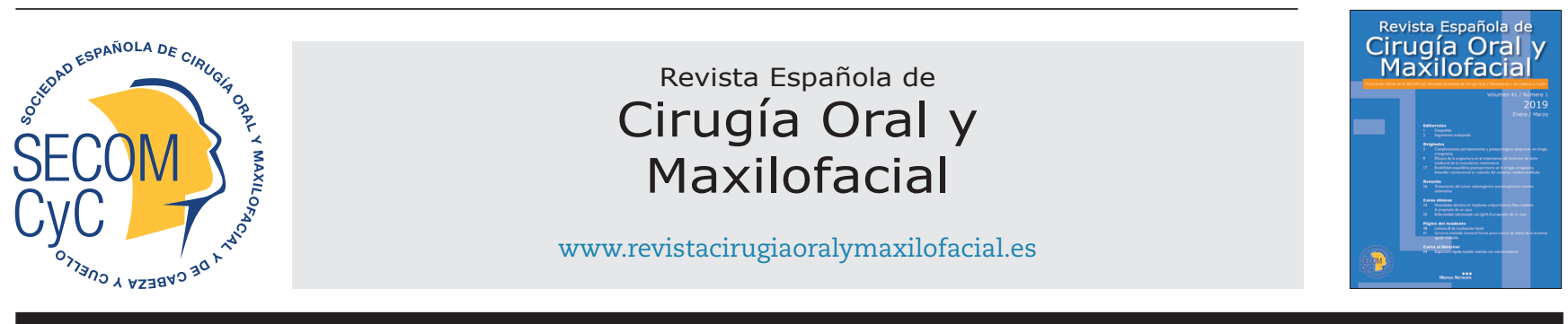

\title{
Original
}

\section{Radioterapia en colgajos peroneos. Revisión de la densidad ósea y complicaciones en colgajos peroneos irradiados frente a no irradiados}

\author{
Francisco Merino Domingo, Marc Espino Segura-Illa, María Mejía Nieto, \\ Víctor Zafra Vallejo, Pablo López Fernández y Gregorio Sánchez Aniceto
}

\author{
Servicio Cirugía Oral y Maxilofacial. Hospital Universitario 12 de Octubre. Madrid. España
}

\section{INFORMACIÓN DEL ARTÍCULO}

Historia del artículo:

Recibido el 21 de junio de 2018

Aceptado el 2 de febrero de 2019

Palabras clave:

Osteoradionecrosis, colgajo peroneo, densidad ósea.

\section{R E S U M E N}

Introducción: El colgajo peroneo es actualmente una de las técnicas más elegidas para la reconstrucción de defectos mandibulares. Muchos de los pacientes reconstruidos con este colgajo presentan patología oncológica que precisa tratamiento adyuvante con radioterapia. Los efectos adversos de la radioterapia sobre los tejidos son conocidos, pudiendo desembocar en osteoradionecrosis. El objetivo de este estudio es el análisis de la densidad ósea de los colgajos peroneos en los pacientes que han recibido radioterapia frente a los que no y su posible correlación con las complicaciones posteriores.

Material y métodos: Realizamos un estudio retrospectivo de los colgajos peroneos realizados por nuestro servicio desde enero 2011 hasta diciembre 2016. Los criterios de inclusión de los pacientes fue el haber sido reconstruidos con un colgajo peroneo y disponer de un TC de control a los tres meses postradioterapia en caso de haberla precisado. Se recogió la edad, densidad ósea peronea, desviación estándar cuerpo mandibular remanente y colgajo peroneo, así como las complicaciones postoperatorias. Los datos extraídos fueron analizados a través del Software SAS (Statistical Analisys System).

Resultados: En total se recogieron 61 pacientes, siendo la media de edad de 54,46 años. De estos 61 pacientes se excluyeron 11 al no presentar TC de control; de los 50 pacientes restantes, 27 recibieron tratamiento radioterápico adyuvante (54\%), mientras que 23 no la recibieron (46\%). En los pacientes irradiados, un alto porcentaje (84,62 \%) recibieron altas dosis de radioterapia (> 60 Gy). Se encontraron diferencias estadísticamente significativas $(p<0,05)$ respecto a la edad y la desviación estándar de la densidad ósea del cuerpo mandibular remanente, mientras que en el resto de los parámetros no se encontraron diferencias estadísticamente significativas. Los pacientes irradiados presentaron un mayor porcentaje de complicaciones frente a los pacientes no irradiados, sin diferencias estadísticamente significativas.

\footnotetext{
*Autor para correspondencia

Correo electrónico: fmerinodomingo@gmail.com (Francisco Merino Domingo).

DOI: 10.20986/recom.2019.1047/2019
}

1130-0558/@ 2019 SECOM. Publicado por Inspira Network. Este es un artículo Open Access bajo la licencia CC BY-NC-ND (http:// creativecommons.org/licenses/by-nc-nd/4.0/). 
Discusión: Las nuevas técnicas de radioterapia de intensidad modulada permiten una dosis relativamente uniforme en un objetivo, evitando altas dosis en los tejidos circuncidantes. A pesar del avance en las técnicas de radioterapia, actualmente en la mayoría de centros se continúa realizando un tratamiento completo del volumen del lecho tumoral, que incluye en el campo de irradiación el colgajo con el que se reconstruye. Los casos de osteonecrosis mandibular que se presentaron fueron en pacientes irradiados y a nivel del cuerpo mandibular remanente. La densidad ósea a nivel del colgajo peroneo sí que presentó diferencias estadísticamente significativas en pacientes irradiados frente a no irradiados; este aspecto puede influir en el índice de complicaciones.

Conclusiones: Consideramos, con los resultados del estudio, plantear nuevos estudios prospectivos para valorar la necesidad de irradiar el tejido óseo del colgajo peroneo como parte del volumen del lecho tumoral, siendo que este tejido no ha estado en contacto con el tumor primario, ante el mayor índice de complicaciones asociados a la radioterapia en estos tejidos.

\section{Effects of radiotherapy in fibular free flaps. Study of bone density and complications in irradiated fibular flaps vs no irradiated}

\section{A B S T R A C T}

Keywords:

Osteoradionecrosis, fibular flap, bone density.
Introduction: The fibular flap is currently one of the most chosen techniques for the reconstruction of mandibular defects. Many of the patients reconstructed with this flap present oncological pathology that requires adjuvant treatment with radiotherapy. The adverse effects of radiotherapy on tissues are known, and can lead to osteoradionecrosis.

The aim of this study is to analyze the bone density of peroneal flaps in patients who have received radiotherapy against those who do not and their possible correlation with subsequent complications.

Material and methods: We designed a retrospective study of the fibular flaps performed by our service from January 2011 to December 2016. The criteria for inclusion of patients was to have been reconstructed with a peroneal flap and have a control CT at 3 months after radiotherapy. Age, bone density, standard deviation and further complications were extracted. The extracted data was analyzed through the SAS (statistical Analisys System) Software.

Results: 61 patients were collected; the mean age was of 54.46 years. Of these 61 patients were excluded 11 by not presenting control TC, of the 50 patients remaining, 27 received adjuvant radiotherapy treatment (54\%) while 23 did not receive (46\%). In irradiated patients a high percentage $(84.62 \%)$ received high doses of radiotherapy (> $60 \mathrm{~Gy}$ ). Statistically significant differences were found $(\mathrm{P}<0.05)$ with respect to age and standard deviation of bone density of the remaining mandibular body, while in the rest of the parameters no statistical significant differences were found. Irradiated patients presented a higher percentage of complications compared to non-irradiated patients without statistically significant differences.

Discussion: The new techniques of intensity-modulated radiotherapy allow a relatively uniform dose in a target, avoiding high doses in the circumcising tissues. Despite the progress in radiotherapy techniques, currently in most centers, it continues to perform a complete treatment of the volume of the tumor bed that includes in the field of irradiation the flap with which it is reconstructed. The cases of mandibular osteonecrosis that were presented were in irradiated patients and in the remaining mandible. Bone density at the level of the fibular flap showed statistically significant differences in irradiated patients compared to non-irradiated, this aspect may influence in the index of complications.

Conclusions: We consider to propose new prospective studies to assess the need to irradiate the bone tissue of the fibular flap as part of the volume of the tumor bed, knowing that this tissue has not been in contact with the primary tumor, and the high index of complications associated with radiotherapy.

\section{INTRODUCCIÓN}

El colgajo peroneo es actualmente una de las técnicas más elegidas para la reconstrucción de defectos mandibulares. Desde su introducción por Hidalgo en $1989^{1}$, el col- gajo vascularizado peroneo se ha convertido en la primera opción para la reconstrucción de defectos óseos mandibulares extensos. El peroné posee la ventaja de poder aportar una extensa cantidad de material óseo con un pedículo vascular largo. 
Muchos de los pacientes reconstruidos con este colgajo presentan patología oncológica que precisa tratamiento adyuvante con radioterapia. Los efectos adversos de la radioterapia sobre los tejidos son bien conocidos ${ }^{2}$, con complicaciones como: mucositis, dermatitis, sobreinfecciones por hongos, alteración de la secreción salivar y alteraciones dentales, pudiendo desembocar en osteoradionecrosis (Figura 1) ${ }^{3}$.

El tratamiento de los objetivos con técnicas de radioterapia de intensidad modulada (IMRT) permite una dosis relativamente uniforme en un objetivo con forma irregular, evitando altas dosis en los tejidos circuncidantes. A pesar del avance en las técnicas de radioterapia, actualmente en la mayoría de los centros se continúa realizando un tratamiento completo del volumen del lecho tumoral, que incluye en el campo de irradiación el colgajo con el que se reconstruye (Figura 2).

El objetivo de este artículo es el análisis de la densidad ósea de los colgajos peroneos en los pacientes que han recibido radioterapia frente a los que no y su posible correlación con las complicaciones posteriores.

\section{MATERIAL Y MÉTODOS}

Realizamos un estudio retrospectivo descriptivo de los colgajos peroneos realizados por el Servicio de Cirugía Oral y Maxilofacial del Hospital Universitario 12 de Octubre desde enero de 2011 hasta diciembre de 2016.

Los criterios de inclusión de los pacientes fueron los siguientes:

- Haber sido reconstruidos con, al menos, un colgajo peroneo.

- Disponer de un TC de control a los tres meses postradioterapia (en caso de haberla precisado). En caso de no precisar radioterapia, disponer de un TC de control a los tres meses de la cirugía.

Se procedió al análisis de las imágenes con el programa Philips Isite Enterprise por parte de dos investigadores. Se pro-

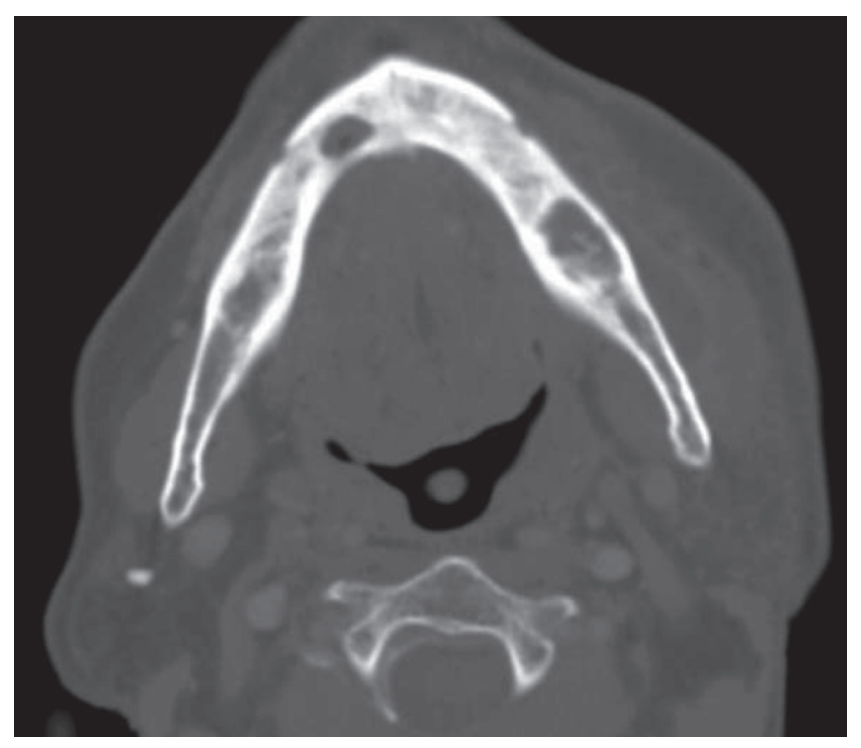

Figura 1. TC en paciente radiado con signos de osteoradionecrosis mandibular establecida. tocolizó el marcaje a nivel del cuerpo mandibular remanente de tres regiones de interés que incluyeran ambas corticales mandibulares (vestibular y lingual), así como la medular ósea (Figura 3A). A nivel del colgajo peroneo se realizaron otras tres mediciones, incluyendo dentro de la región de interés ambas corticales peroneas, así como la medular (Figura 3B). De cada región de interés se sacó la medición de la densidad ósea en unidades Hounsfield (UH), así como su desviación estándar entendiéndola como la desviación que presentaron los datos en su distribución respecto de la media.

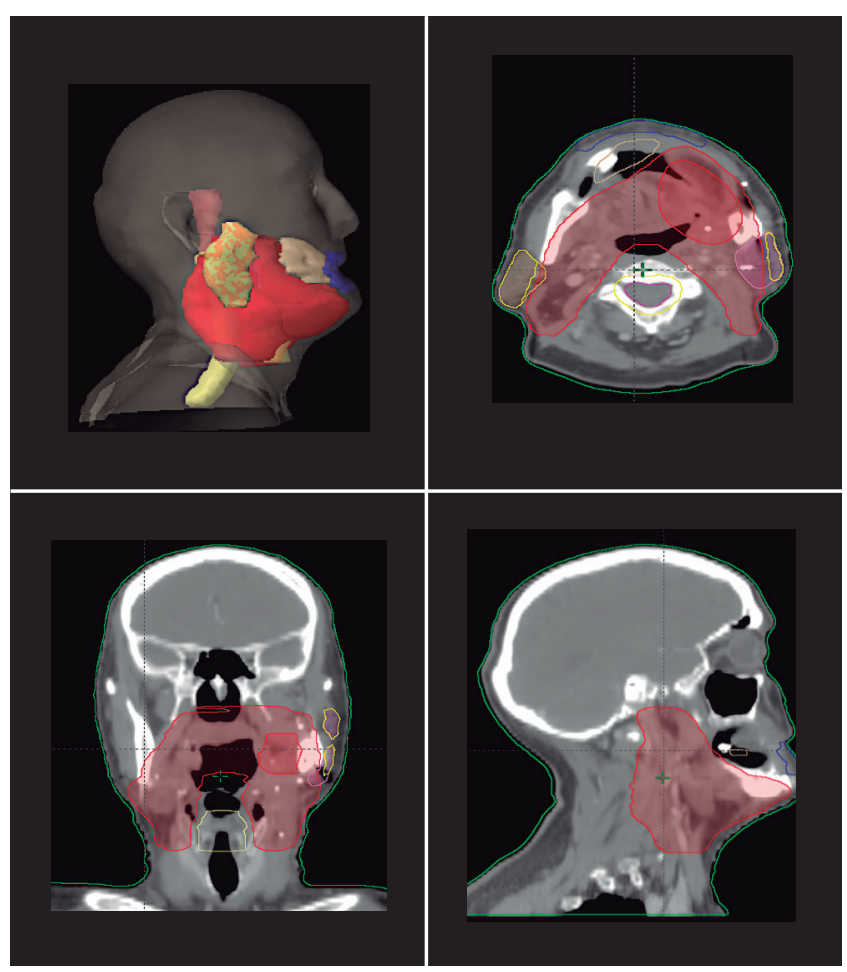

Figura 2. Planificación tridimensional de tratamiento radioterápico adyuvante en paciente con carcinoma epidermoide de reborde alveolar.

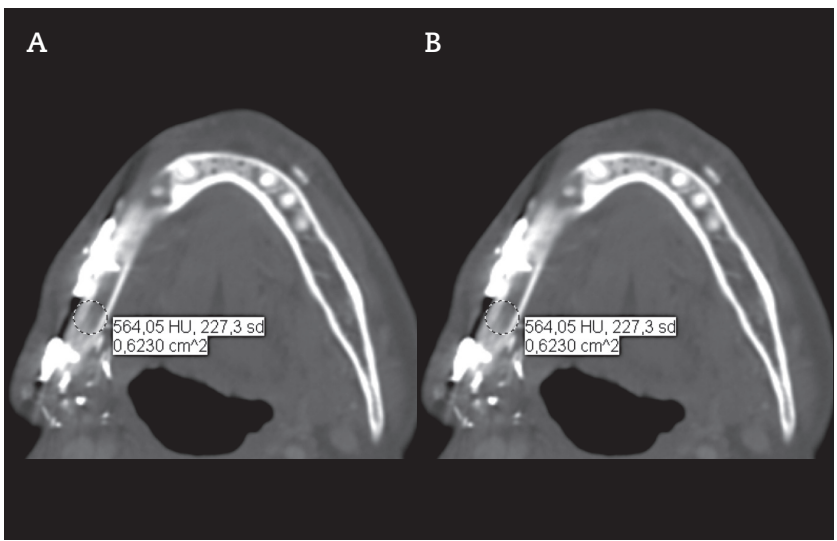

Figura 3. A. Marcaje de ROI para medición de la densidad ósea en la mandíbula remanente. B. Marcaje de ROI para medición de la densidad ósea en el colgajo peroneo. 
Se recogieron las complicaciones postoperatorias asociadas a la zona receptora del colgajo, incluyendo: osteoradionecrosis, pseudoartrosis, exposición/fallo material de osteosíntesis y fístula cutánea. Es conocido que el índice de complicaciones y el tipo de complicaciones está asociado también a la situación general preoperatoria del paciente, así como a la patología primaria que puede influir en el éxito de la reconstrucción, sabiendo que esto puede suponer un sesgo de selección.

Los datos extraídos fueron analizados a través del Software SAS (Statistical Analisys System). Se utilizó para comparar las muestras el test no paramétrico de Wilcoxon, al no seguir nuestra muestra una distribución normal, donde la significación estadística fue $p<0,05$.

\section{RESULTADOS}

En total se recogieron 61 pacientes, siendo la media de edad de 54,46 años. De estos 61 pacientes, se excluyeron 11 al no presentar TC de control. De los restantes, 33 (66,00 \%) fueron hombres y 17 (34,00\%) fueron mujeres.

La causa principal de reconstrucción con colgajo peroneo fue la presencia de patología tumoral maligna (34 casos), seguido de tumores benignos óseos (5 casos), necrosis mandibular (5 casos), recidivas oncológicas (3 casos), secuelas por arma de fuego (2 casos) y fisura labio-palatina (1 caso).
De los pacientes oncológicos, un elevado porcentaje $(82,36 \%)$ presentaron un estadio avanzado de la enfermedad (Tabla I). De los 50 pacientes, 27 recibieron tratamiento radioterápico adyuvante (54\%) mientras que 23 no lo recibieron (46\%). En los pacientes irradiados, un alto porcentaje $(84,62 \%)$ recibieron altas dosis de radioterapia (>60 Gy). En el análisis de los datos en función de si habían recibido RT o no, se estudiaron como variables independientes: la edad, la densidad ósea del cuerpo mandibular remanente, la desviación estándar de la densidad ósea del cuerpo mandibular remanente, la densidad ósea del colgajo peroneo y la desviación estándar de la densidad ósea del colgajo peroneo (Tablas II a IV).

Los pacientes irradiados presentaron una media de edad de 58,81 años frente a los 47,96 años del grupo no irradiado.

\begin{tabular}{ccccc} 
Tabla I. & \multicolumn{5}{c}{ Distribución según estadios tumorales } \\
Estadio & Frecuencia & Porcentaje & $\begin{array}{c}\text { Frecuencia } \\
\text { acumulada }\end{array}$ & $\begin{array}{c}\text { Porcentaje } \\
\text { acumulado }\end{array}$ \\
\hline I & 4 & 11,76 & 4 & 11,76 \\
II & 2 & 5,88 & 6 & 17,65 \\
III & 5 & 14,71 & 11 & 32,35 \\
IVa & 22 & 64,71 & 33 & 97,06 \\
IVc & 1 & 2,94 & 34 & 100,00 \\
\hline
\end{tabular}

\begin{tabular}{|c|c|c|c|c|c|c|}
\hline Variable & Media & DS & Mínimo & Máximo & $\mathrm{N}$ & $\begin{array}{c}\text { Número de } \\
\text { valores ausentes }\end{array}$ \\
\hline Edad & 53,26 & 16,00 & 21,00 & 80,00 & 50 & 0 \\
\hline Mandíbula & 655,47 & 181,71 & 190,03 & 956,42 & 45 & 5 \\
\hline Mandíbula DS & 392,96 & 111,77 & 105,77 & 723,67 & 45 & 5 \\
\hline Peroné & 671,50 & 205,17 & 277,12 & 1293,40 & 50 & 0 \\
\hline Peroné DS & 420,52 & 153,09 & 126,50 & 809,77 & 50 & 0 \\
\hline
\end{tabular}

Tabla III. Datos pacientes SIN radioterapia postoperatoria (DS: desviación estándar)

\begin{tabular}{lcccccc}
\multicolumn{1}{c}{ Variable } & Media & DS & Mínimo & Máximo & N & $\begin{array}{c}\text { Número de } \\
\text { valores ausentes }\end{array}$ \\
\hline Edad & 47,96 & 17,92 & 21,00 & 79,00 & 23 & 0 \\
Mandíbula & 645,33 & 193,90 & 338,67 & 956,42 & 19 & 4 \\
Mandíbula DS & 427,49 & 104,46 & 274,03 & 697,00 & 19 & 23 \\
Peroné & 709,49 & 228,87 & 292,80 & 1293,40 & 23 & 0 \\
Peroné DS & 462,60 & 174,25 & 230,57 & 797,50 & 0 \\
\hline
\end{tabular}

Tabla IV. Datos pacientes CON radioterapia postoperatoria (DS: desviación estándar)

\begin{tabular}{lcccccc}
\multicolumn{1}{c}{ Variable } & Media & DS & Mínimo & Máximo & N & $\begin{array}{c}\text { Número de } \\
\text { valores ausentes }\end{array}$ \\
\hline Edad & 58,81 & 11,89 & 34,00 & 80,00 & 941,88 & 26 \\
Mandíbula & 654,89 & 174,54 & 190,03 & 491,17 & 25 & 25 \\
Mandíbula DS & 353,49 & 87,14 & 105,77 & 994,20 & 26 & 1 \\
Peroné & 637,29 & 183,99 & 277,12 & 548,17 & 26 & 0 \\
Peroné DS & 368,31 & 93,24 & 126,50 & & 0 \\
\hline
\end{tabular}


La media de la densidad ósea del cuerpo mandibular remanente en el paciente irradiado fue de 654,89 por 645, $33 \mathrm{UH}$ de los pacientes no irradiados. La media de la densidad ósea de la porción ósea del colgajo peroneo fue de 637,29 frente a 709,49 UH de los pacientes no irradiados.

Se encontraron diferencias estadísticamente significativas $(\mathrm{p}<0,05)$ respecto a la edad y la densidad de la porción ósea del colgajo peroneo, mientras que en el resto de los parámetros estudiados no se encontraron diferencias estadísticamente significativas (Figura 4).

Aproximadamente, la mitad de los pacientes (47,82\%) presentaron algún tipo de complicación. La complicación más frecuente fue la exposición de material de osteosíntesis, seguida por la fístula cutánea. Los pacientes irradiados presentaron
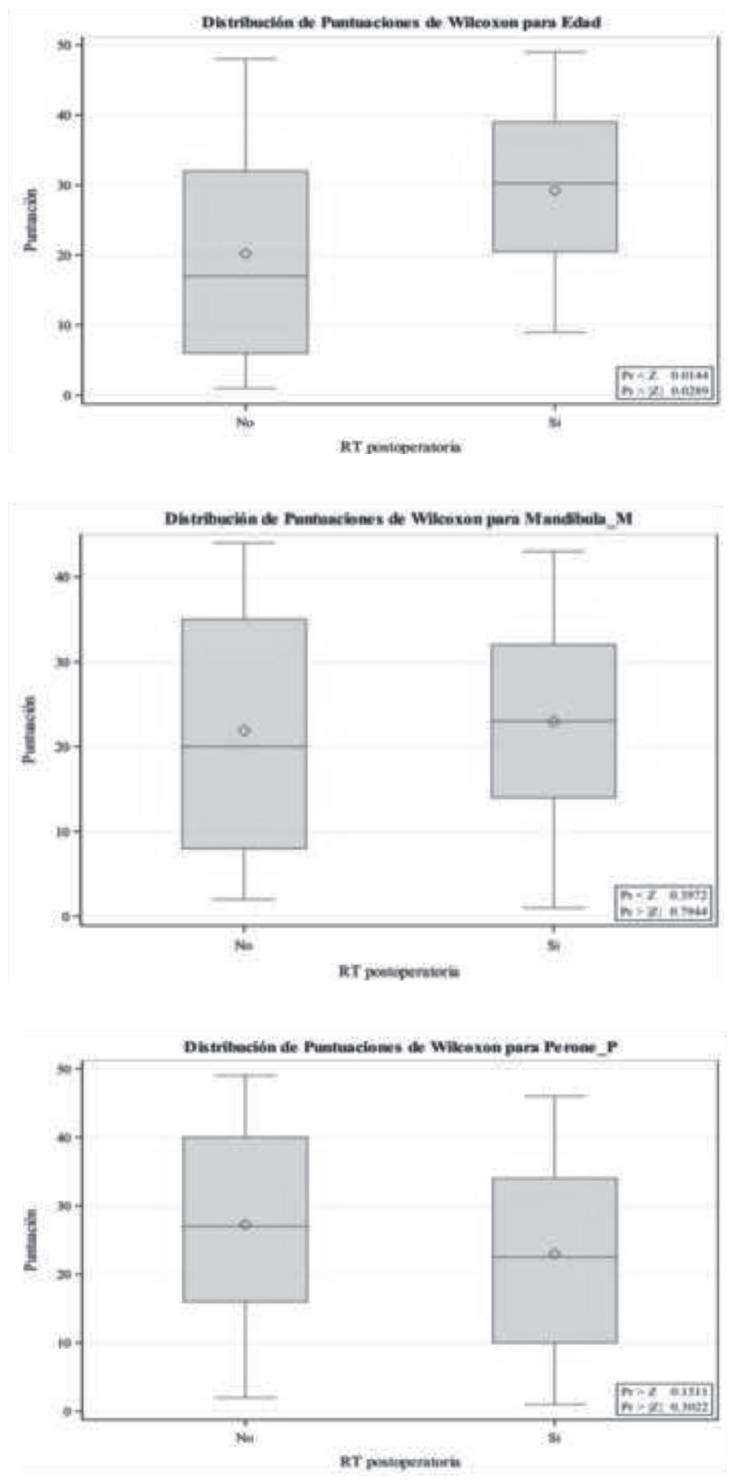

Figura 4. Prueba de rangos de Wilcoxon para edad, peroné y mandíbula remanente. un mayor porcentaje de complicaciones (51,85\%) frente a los pacientes no irradiados $(34,79 \%)$.

Dentro de los pacientes irradiados presentaron exposición del material de osteosíntesis un 18,52 \%, seguido por fístula cutánea (18,52\%), pseudoartrosis (3,7\%) y osteonecrosis (7,4 \%). Los casos de osteonecrosis se presentaron a nivel de la mandíbula remanente.

En los pacientes no irradiados la complicación más frecuente fue también la exposición de material de osteosíntesis (21,74\%), seguido de fístula cutánea (4,35\%). Las diferencias respecto a las complicaciones entre pacientes irradiados y no irradiados no fueron estadísticamente significativas $(p<0,05)$ (Tabla V).

\section{DISCUSIÓN}

El colgajo peroneo es una de las técnicas más utilizadas para la reconstrucción de defectos óseos a nivel facial. Desde su introducción por Hidalgo para reconstrucción de defectos mandibulares, ha ido ganando popularidad hasta convertirse en el tratamiento gold stándar para la reconstrucción de una gran variedad de defectos.

Una de las principales indicaciones de utilización de este colgajo es la reconstrucción, en un mismo tiempo o diferido, de pacientes con patología oncológica. Un alto porcentaje de estos pacientes precisarán tratamiento radioterápico adyuvante posterior secundario a su patología oncológica. La indicación de radioterapia postoperatoria ha aumentado la supervivencia tanto global como libre de enfermedad de estos pacientes, disminuyendo la tasa de recidivas locorregionales, pero es conocido que la radioterapia en cabeza y cuello presenta varios efectos adversos que pueden desembocar en importantes complicaciones, como la osteoradionecrosis. La osteoradionecrosis es una complicación de difícil manejo y con un importante efecto sobre la calidad de vida del paciente.

Las técnicas de radioterapia han evolucionado, siendo hoy en día el tratamiento de los objetivos con técnicas de radioterapia de intensidad modulada (IMRT) la técnica más habitual. Esto permite una dosis relativamente uniforme en un objetivo con forma irregular, evitando altas dosis en los tejidos circundantes. Las dianas sobre las que se dirige la radioterapia en pacientes intervenidos son el lecho primario, donde se encontraba el tumor, así como su vía de diseminación habitual (en caso del cáncer de cabeza y cuello las cadenas linfáticas cervicales). A pesar de realizar una planificación tridimensional del volumen a irradiar, hoy en día la mayoría de centros continúa irradiando el tejido óseo del colgajo peroneo como parte del volumen del lecho tumoral, siendo que este tejido no ha estado en contacto con el tumor primario.

El objetivo de este artículo es valorar que efecto tiene la radioterapia sobre la densidad ósea del colgajo; para ello, basándonos en otros artículos ${ }^{4}$, decidimos cuantificar la densidad ósea a través de la realización de un TC, entendiendo esta alteración como un factor predisponente para el desarrollo de necrosis mandibular ${ }^{5}$.

Recientemente, Kraeima y cols. ${ }^{6}$ plantean la utilización de la planificación tridimensional IMRT para guiar la resección en el tratamiento de la osteoradionecrosis al área que haya recibido una mayor dosis radioterápica. 
Tabla V. Complicaciones en pacientes con y sin RT postoperatoria

\begin{tabular}{|c|c|c|c|c|c|c|c|}
\hline \multirow{2}{*}{$\begin{array}{c}\text { RT } \\
\text { Postoperatoria }\end{array}$} & \multicolumn{7}{|c|}{ Complicaciones } \\
\hline & $\begin{array}{c}\text { Sin } \\
\text { complicaciones }\end{array}$ & ORN & Pseudoartrosis & $\begin{array}{l}\text { Exposición M. } \\
\text { Osteosíntesis }\end{array}$ & Fístula & Otros & Total \\
\hline \multicolumn{8}{|l|}{ NO } \\
\hline Frecuencia & 15 & 0 & 0 & 5 & 1 & 2 & 23 \\
\hline Porcentaje & 30 & 0 & 0 & 10 & 2 & 4 & 46 \\
\hline Pct fila & 65,21 & 0 & 0 & 21,74 & 4,35 & 8,7 & \\
\hline $\begin{array}{l}\text { Pct columna } \\
\text { Sí }\end{array}$ & 53,57 & 0 & 0 & 50 & 16,66 & 66,6 & \\
\hline Frecuencia & 13 & 2 & 1 & 5 & 5 & 1 & 27 \\
\hline Porcentaje & 26 & 4 & 2 & 10 & 10 & 2 & 54 \\
\hline Pct fila & 48,15 & 7,4 & 3,7 & 18,52 & 18,52 & 3,7 & \\
\hline Pct columna & 46,42 & 100 & 100 & 50 & 83,34 & 33,3 & \\
\hline Total & 28 & 2 & 1 & 10 & 6 & 3 & 50 \\
\hline Porcentaje & 56 & 4 & 2 & 20 & 12 & 6 & 100 \\
\hline
\end{tabular}

Revisando nuestros resultados encontramos diferencias estadísticamente significativas tanto en la edad de los pacientes como en la densidad de la porción ósea del colgajo peroneo. Las diferencias en la densidad ósea según la edad no solo se asocian a que el hueso a medida que envejece se transforma en hueso más osteoporótico, conllevando una menor densidad ósea, sino que también los pacientes más jóvenes, en nuestra serie, tuvieron menor frecuencia de patología tumoral maligna, por lo que fue menor el número de pacientes que precisaron tratamiento radioterápico adyuvante.

Respecto a la densidad ósea del cuerpo mandibular remanente no se encontraron diferencias estadísticamente significativas, pero los casos de osteonecrosis mandibular que se presentaron fueron en pacientes irradiados y a nivel del cuerpo mandibular remanente, lo cual convendría revisar si realmente el volumen tratado con radioterapia se limitaba al volumen tumoral con márgenes, o bien se ampliaba, tratando parte de la mandíbula remanente.

La densidad ósea a nivel del colgajo peroneo sí que presentó diferencias estadísticamente significativas en pacientes irradiados frente a no irradiados; este aspecto puede influir en el índice de complicaciones, así como en la futura osteointegración de los implantes dentales para la rehabilitación dental.

Los pacientes irradiados presentaron un mayor porcentaje de complicaciones postoperatorias que los pacientes no irradiados, sin diferencias estadísticamente significativas. Chen y cols. ${ }^{7}$ ya concluyeron en su estudio que el tabaco y la radioterapia eran predictores independientes de complicaciones tardías en pacientes reconstruidos con colgajos peroneos microvascularizados $^{7}$. Es conocido que las complicaciones asociadas a estos pacientes en numerosos casos pueden deberse a las comorbilidades de los mismos y a su patología primaria, siendo más frecuente las complicaciones en pacientes con patología maligna que patología benigna, por lo que no se puede descartar que exista un sesgo de selección.

En nuestro estudio, la complicación más frecuente fue en ambos casos la exposición del material de osteosíntesis, aunque la frecuencia fue mucho mayor en los pacientes irradiados. La fístula cutánea fue la siguiente complicación más frecuente en ambos grupos. La osteonecrosis apareció únicamente en los pacientes irradiados, siendo un 7,4 \% de las complicaciones en los pacientes irradiados. La pseudoartrosis también presentó mayor frecuencia en el grupo de pacientes irradiados.

La presencia de alteraciones en la densidad ósea del colgajo peroneo, un mayor porcentaje de complicaciones en los pacientes irradiados, así como los importantes avances en las técnicas de radioterapia, hace replantearnos la necesidad de irradiar a dosis altas (> $60 \mathrm{~Gy}$ ) la porción ósea del colgajo peroneo. Los autores consideramos que se precisan estudios prospectivos con una mayor cohorte para responder a esta pregunta.

\section{CONCLUSIONES}

Las nuevas técnicas de radioterapia de intensidad modulada permiten una dosis relativamente uniforme en un objetivo, evitando altas dosis en los tejidos circuncidantes. En nuestro trabajo encontramos diferencias significativas en la edad en la que se habían radiado los pacientes, lo cual parece estar asociado al mayor porcentaje de benignidad de la patología a menor edad del paciente que presentó nuestra muestra. Los valores de densidad ósea obtuvieron diferencias estadísticamente significativas $(p<0,05)$ a nivel peroneo en los pacientes irradiados frente a los que no precisaron radioterapia. A pesar de no presentar diferencias estadísticamente significativas, los pacientes irradiados presentaron un mayor porcentaje de complicaciones postoperatorias, lo cual, bajo nuestro criterio, plantea si con las técnicas actuales de radioterapia es necesario irradiar a dosis altas la porción ósea del colgajo, planteando un mayor trabajo conjunto del cirujano con el oncólogo radioterapeuta en la delimitación del volumen tumoral a tratar.

\section{CONFLICTOS DE INTERÉS}

No existen conflictos de interés. 


\section{FINANCIACIÓN}

Los autores no han recibido financiación para realizar este estudio.

\section{B I B L I O G R A F Í A}

1. Hidalgo DA. Fibula free flap: a new method of mandible reconstruction. Plast Reconstr Surg. 1989;84(1):71-9.

2. Benatar MJ, Dassonville O, Chamorey E, Poissonnet G, Ettaiche $\mathrm{M}$, Pierre CS, Benezery $\mathrm{K}$, et al. Impact of preoperative radiotherapy on head and neck free flap reconstruction: A report on 429 cases. J Plast Reconstr Aesthet Surg. 2013;66(4):478-82. DOI: 10.1016/j.bjps.2012.12.019.

3. Marx RE, Johnson RP. Studies in the radiobiology of osteoradionecrosis and their clinical significance. Oral Surg Oral Med Oral Pathol. 1987;64(4):379-90.
4. Koulalias V, Liakouli Z, Zygogianni A, Mystakidou K, Kouvaris JR. Bone Density as a Marker of Response to Radiotherapy in Bone Metastatic Lesions: A Review of the Published Data. Int J Mol Sci. 2016;17(9):1931. DOI: 10.3390/ijms17091391.

5. Monson L, Lin Jing X, Donneys A, Farberg AS, Buchman SR. Doseresponse effect of human equivalent radiation in the murine mandible: A radiomorphometric assessment. J Craniofac Surg. 2013;24(5):1593-8. DOI: 10.1097/SCS.0b013e31826cfeea.

6. Kraiema J, Steenbakkers RJHM, Spijkervet FKL, Roodenburg JLN, Witjes MJH. Secondary surgical management of osteoradionecrosis using three-dimensional isodose curve visualization: a report of three cases. Int J Oral Maxillofac Surg. 2018;47(2):214-9. DOI: 10.1016/j.ijom.2017.08.002.

7. Chen Y, Wu J, Gokavarapu S, Shen Q, Ji T. Radiotherapy and Smoking History Are Significant Independent Predictors for Osteosynthesis-Associated Late Complications in Vascular Free Fibula Reconstruction Mandible. J Craniofac Surg. 2017;28(6):1508-13. DOI: 10.1097/SCS.0000000000003704. 